\title{
On Algebraic Semigroups and Monoids, II
}

\author{
Michel Brion
}

\begin{abstract}
Consider an algebraic semigroup $S$ and its closed subscheme of idempotents, $E(S)$. When $S$ is commutative, we show that $E(S)$ is finite and reduced; if in addition $S$ is irreducible, then $E(S)$ is contained in a smallest closed irreducible subsemigroup of $S$, and this subsemigroup is an affine toric variety. It follows that $E(S)$ (viewed as a partially ordered set) is the set of faces of a rational polyhedral convex cone. On the other hand, when $S$ is an irreducible algebraic monoid, we show that $E(S)$ is smooth, and its connected components are conjugacy classes of the unit group.
\end{abstract}

\section{Introduction}

This article continues the study of algebraic semigroups and monoids (not necessarily linear), began in [Ri98, Ri07] for monoids and in [Br12, BrRe12] for semigroups. The idempotents play an essential rôle in the structure of abstract semigroups; by results of [loc. cit.], the idempotents of algebraic semigroups satisfy remarkable existence and finiteness properties. In this article, we consider the subscheme of idempotents, $E(S)$, of an algebraic semigroup $S$ over an algebraically closed field; we show that $E(S)$ has a very special structure under additional assumptions on $S$. Our first main result states:

Theorem 1.1. Let $M$ be an irreducible algebraic monoid, and $G$ its unit group. Then the scheme $E(M)$ is smooth, and its connected components are conjugacy classes of $G$.

Note that the scheme of idempotents of an algebraic semigroup is not necessarily smooth. Consider indeed an arbitrary variety $X$ equipped with the composition law $(x, y) \mapsto x$; then $X$ is an algebraic semigroup, and $E(X)$ is the whole $X$. Yet the scheme of idempotents is reduced for all examples that we know of; it is tempting to conjecture that $E(S)$ is reduced for any algebraic semigroup $S$.

When $S$ is commutative, $E(S)$ turns out to be a combinatorial object, as shown by our second main result:

Theorem 1.2. Let $S$ be a commutative algebraic semigroup. Then the scheme $E(S)$ is finite and reduced. If $S$ is irreducible, then $E(S)$ is contained in a smallest closed irreducible subsemigroup of $S$; moreover, this subsemigroup is a toric monoid.

By a toric monoid, we mean an irreducible algebraic monoid $M$ with unit group being a torus; then $M$ is affine, as follows e.g. from [Ri07, Thm. 2]. Thus, $M$ may be viewed as an 
affine toric variety (not necessarily normal). Conversely, every such variety has a unique structure of algebraic monoid that extends the multiplication of its open torus (see e.g. [Ri98, Prop. 1]). So we may identify the toric monoids with the affine toric varieties. Toric monoids have been studied by Putcha under the name of connected diagonal monoids (see [Pu81]); they have also been investigated by Neeb in [Ne92].

In view of Theorem 1.2 and of the structure of toric monoids, the set of idempotents of any irreducible commutative algebraic semigroup, equipped with its natural partial order, is isomorphic to the poset of faces of a rational polyhedral convex cone.

Theorem 1.2 extends readily to the case where $S$ has a dense subsemigroup generated by a single element; then $S$ is commutative, but not necessarily irreducible. Thereby, one associates an affine toric variety with any point of an algebraic semigroup; the corresponding combinatorial data may be seen as weak analogues of the spectrum of a linear operator (see Example 3.9 for details). This construction might deserve further study.

This article is organized as follows. In Subsection 2.1, we present simple proofs of some basic results, first obtained in [Br12, BrRe12] by more complicated arguments; also, we prove the first assertion of Theorem 1.2. Subsection 2.2 investigates the local structure of an algebraic semigroup at an idempotent, in analogy with the Peirce decomposition,

$$
R=e \operatorname{Re} \oplus(1-e) \operatorname{Re} \oplus e R(1-e) \oplus(1-e) R(1-e),
$$

of a ring $R$ equipped with an idempotent $e$. As an application, we show that the isolated idempotents of an irreducible algebraic semigroup are exactly the central idempotents (Proposition 2.10). In Subsection 2.3, we obtain a slightly stronger version of Theorem 1.1, by combining our local structure analysis with results of Putcha on irreducible linear algebraic monoids (see [Pu88, Chap. 6]). As an application, we generalize Theorem 1.1 to the intervals in $E(S)$, where $S$ is an irreducible algebraic semigroup in characteristic zero (Corollary 2.17).

We return to commutative semigroups in Subsection 3.1, and show that every irreducible commutative algebraic semigroup has a largest closed toric submonoid (Proposition 3.2). The structure of toric monoids is recalled in Subsection 3.2, and Theorem [1.2 is proved in the case of such monoids. The general case is deduced in Subsection 3.3, which also contains applications to algebraic semigroups having a dense cyclic subsemigroup (Corollary 3.7).

In the final Subsection 3.4, we consider those irreducible algebraic semigroups $S$ such that $E(S)$ is finite. We first show how to reduce their structure to the case where $S$ is a monoid and has a zero; then $S$ is linear in view of [BrRi07, Cor. 3.3]). Then we present another proof of a result of Putcha: any irreducible algebraic monoid having a zero and finitely many idempotents must have a solvable unit group (see [Pu82, Cor. 10], and [Pu88, Prop. 6.24] for a generalization). Putcha also showed that any irreducible linear algebraic monoid with nilpotent unit group has finitely many idempotents, but this does not extend to solvable unit groups (see [Pu81, Thm. 1.12, Ex. 1.15]). We refer to work of Huang (see [Hu96a, Hu96b]) for further results on irreducible linear algebraic monoids having finitely many idempotents. 
Notation and conventions. Throughout this article, we consider varieties and schemes over a fixed algebraically closed field $k$. We use the textbook [Ha77] as a general reference for algebraic geometry. Unless otherwise stated, schemes are assumed to be separated and of finite type over $k$; a variety is a reduced scheme (in particular, varieties are not necessarily irreducible). By a point of a variety $X$, we mean a $k$-rational point; we identify $X$ with its set of points equipped with the Zariski topology and with the structure sheaf.

An algebraic semigroup is a variety $S$ equipped with an associative composition law $\mu: S \times S \rightarrow S$. For simplicity, we denote $\mu(x, y)$ by $x y$ for any $x, y \in S$. A point $e \in S$ is idempotent if $e^{2}=e$. The set of idempotents is equipped with a partial order $\leq$ defined by $e \leq f$ if $e=e f=f e$. Also, the idempotents are the $k$-rational points of a closed subscheme of $S$ : the scheme-theoretic preimage of the diagonal under the morphism $S \rightarrow S \times S, x \mapsto\left(x^{2}, x\right)$. We denote that subscheme by $E(S)$.

An algebraic monoid is an algebraic semigroup $M$ having a neutral element, $1_{M}$. The unit group of $M$ is the subgroup of invertible elements, $G(M)$; this is an algebraic group, open in $M$ (see [Ri98, Thm. 1] in the case where $M$ is irreducible; the general case follows easily, see [Br12, Thm. 1]).

We shall address some rationality questions for algebraic semigroups, and use Sp98, Chap. 11] as a general reference for basic rationality results on varieties. As in [loc. cit.], we fix a subfield $F$ of $k$, and denote by $F_{s}$ the separable closure of $F$ in $k$; the Galois group of $F_{s}$ over $F$ is denoted by $\Gamma$. We say that an algebraic semigroup $S$ is defined over $F$, if the variety $S$ and the morphism $\mu: S \times S \rightarrow S$ are both defined over $F$.

\section{The idempotents of an algebraic semigroup}

\section{$2.1 \quad$ Existence}

We first obtain a simple proof of the following basic result ([Br12, Prop. 1], proved there by reducing to a finite field):

Proposition 2.1. Let $S$ be an algebraic semigroup. Then $S$ has an idempotent.

Proof. Arguing by noetherian induction, we may assume that $S$ has no proper closed subsemigroup. As a consequence, the set of powers $x^{n}$, where $n \geq 1$, is dense in $S$ for any $x \in S$; in particular, $S$ is commutative. Also, $y S$ is dense in $S$ for any $y \in S$; since $y S$ is constructible, it contains a nonempty open subset of $S$. Thus, there exists $n=n(x, y) \geq 1$ such that $x^{n} \in y S$.

Choose $x \in S$. For any $n \geq 1$, let

$$
S_{n}:=\left\{y \in S \mid x^{n} \in y S\right\}
$$

Then each $S_{n}$ is a constructible subset of $S$, since $S_{n}$ is the image of the closed subset $\left\{(y, z) \in S \times S \mid y z=x^{n}\right\}$ under the first projection. Moreover, $S=\bigcup_{n>1} S_{n}$.

To show that the closed subscheme $E(S)$ is nonempty, we may replace $k$ with any larger algebraically closed field, and hence assume that $k$ is uncountable. Then, by the next lemma, there exists $n \geq 1$ such that $S_{n}$ contains a nonempty open subset of $S$. Since the set of powers $x^{m n}$, where $m \geq 1$, is dense in $S$, it follows that $S_{n}$ contains some $x^{m n}$. 
Equivalently, there exists $z \in S$ such that $x^{n}=x^{m n} z$. Let $y:=x^{n}$, then $y=y^{m} z$ and hence $y^{m-1}=y^{2 m-2} z$. Thus, $y^{m-1} z$ is idempotent.

Lemma 2.2. Let $X$ be a variety, and $\left(X_{i}\right)_{i \in I}$ a countable family of constructible subsets such that $X=\bigcup_{i \in I} X_{i}$. If $k$ is uncountable, then some $X_{i}$ contains a nonempty open subset of $X$.

Proof. Since each $X_{i}$ is constructible, it can be written as a finite disjoint union of irreducible locally closed subsets. We may thus assume that each $X_{i}$ is locally closed and irreducible; then we may replace $X_{i}$ with its closure, and thus assume that all the $X_{i}$ are closed and irreducible. We may also replace $X$ with any nonempty open subset $U$, and $X_{i}$ with $X_{i} \cap U$. Thus, we may assume in addition that $X$ is irreducible. We then have to show that $X_{i}=X$ for some $i \in I$.

We now argue by induction on the dimension of $X$. If $\operatorname{dim}(X)=1$, then each $X_{i}$ is either a finite subset or the whole $X$. But the $X_{i}$ cannot all be finite: otherwise, $X$, and hence $k$, would be countable. This yields the desired statement.

In the general case, assume that each $X_{i}$ is a proper subset of $X$. Since the set of irreducible hypersurfaces in $X$ is uncountable, there exists such a hypersurface $Y$ which is not contained in any $X_{i}$. In other words, each $Y \cap X_{i}$ is a proper subset of $Y$. Since $Y=\bigcup_{i \in I} Y \cap X_{i}$, applying the induction assumption to $Y$ yields a contradiction.

Next, we obtain refinements of [Br12, Prop. 4 (iii), Prop. 17 (ii)], thereby proving the first assertion of Theorem 1.2.

Proposition 2.3. Let $S$ be a commutative algebraic semigroup.

(i) The scheme $E(S)$ is finite and reduced.

(ii) $S$ has a smallest idempotent, $e_{0}$.

(iii) If the algebraic semigroup $S$ is defined over $F$, then so is $e_{0}$.

Proof. (i) It suffices to show that the Zariski tangent space $T_{e}(E(S))$ is zero for any idempotent $e$. Since $E(S)=\left\{x \in S \mid x^{2}=x\right\}$ and $S$ is commutative, we obtain

$$
T_{e}(E(S))=\left\{z \in T_{e}(S) \mid 2 f(z)=z\right\}
$$

where $f$ denotes the tangent map at $e$ of the multiplication by $e$ in $S$ (see Lemma 2.5 below for details on the determination of $T_{e}(E(S))$ when $S$ is not necessarily commutative). Moreover, $f$ is an idempotent endomorphism of the vector space $T_{e}(S)$, and hence is diagonalizable with eigenvalues 0 and 1 . This yields the desired vanishing of $T_{e}(E(S))$.

(ii) By (i), the subscheme $E(S)$ consists of finitely many points $e_{1}, \ldots, e_{n}$ of $S$. Their product, $e_{1} \cdots e_{n}=: e_{0}$, satisfies $e_{0}^{2}=e_{0}$ and $e_{0} e_{i}=e_{0}$ for $i=1, \ldots, n$. Thus, $e_{0}$ is the smallest idempotent.

(iii) Assume that the variety $S$ and the morphism $\mu$ are defined over $F$; then $E(S)$ is a $F$-subscheme of $S$, and hence a smooth $F$-subvariety by (i). In view of [Sp98, Thm. 11.2.7], it follows that $E(S)$ (regarded as a finite subset of $S(k)$ ) is contained in $S\left(F_{s}\right)$; also, $E(S)$ is clearly stable by $\Gamma$. Thus, $e_{0} \in S\left(F_{s}\right)$ is invariant under $\Gamma$, and hence $e_{0} \in S(F)$.

We now deduce from Proposition 2.3 another fundamental existence result (which also follows from [BrRe12, Thm. 1]): 
Corollary 2.4. Let $S$ be an algebraic semigroup defined over $F$. If $S$ has an F-rational point, then it has an F-rational idempotent.

Proof. Let $x \in S(F)$ and denote by $\langle x\rangle$ the smallest closed subsemigroup of $S$ containing $x$. Then $\langle x\rangle$ is the closure of the set of powers $x^{n}$, where $n \geq 1$. Thus, $\langle x\rangle$ is a commutative algebraic semigroup, defined over $F$. So $\langle x\rangle$ contains an idempotent defined over $F$, by the previous proposition.

\subsection{Local structure}

In this subsection, we fix an algebraic semigroup $S$ and an idempotent $e \in S$. Then $e$ defines two endomorphisms of the variety $S$ : the left multiplication, $e_{\ell}: x \mapsto e x$, and the right multiplication, $e_{r}: x \mapsto x e$. Clearly, these endomorphisms are commuting idempotents, i.e., they satisfy $e_{\ell}^{2}=e_{\ell}, e_{r}^{2}=e_{r}$, and $e_{\ell} e_{r}=e_{r} e_{\ell}$. Since $e_{\ell}$ and $e_{r}$ fix the point $e$, their tangent maps at that point are commuting idempotent endomorphisms, $f_{\ell}$ and $f_{r}$, of the Zariski tangent space $T_{e}(S)$. Thus, we have a decomposition into joint eigenspaces

$$
T_{e}(S)=T_{e}(S)_{0,0} \oplus T_{e}(S)_{1,0} \oplus T_{e}(S)_{0,1} \oplus T_{e}(S)_{1,1}
$$

where we set

$$
T_{e}(S)_{a, b}:=\left\{z \in T_{e}(S) \mid f_{\ell}(z)=a z, f_{r}(z)=b z\right\}
$$

for $a, b=0,1$. The Zariski tangent space of $E(S)$ at $e$ has a simple description in terms of these eigenspaces:

Lemma 2.5. With the above notation, we have

$$
T_{e}(E(S))=T_{e}(S)_{1,0} \oplus T_{e}(S)_{0,1} .
$$

Moreover, $T_{e}(E(S))$ is the image of $f_{r}-f_{\ell}$.

Proof. We claim that

$$
T_{e}(E(S))=\left\{z \in T_{e}(S) \mid f_{\ell}(z)+f_{r}(z)=z\right\} .
$$

Indeed, recall that $E(S)$ is the preimage of the diagonal under the morphism $S \rightarrow S \times S$, $x \mapsto(\operatorname{sq}(x), x)$, where sq $: S \rightarrow S, x \mapsto x^{2}$ denotes the square map. Thus, we have

$$
T_{e}(E(S))=\left\{z \in T_{e}(S) \mid T_{e}(\mathrm{sq})(z)=z\right\},
$$

where $T_{e}(\mathrm{sq})$ denotes the tangent map of sq at $e$. Also, sq is the composition of the diagonal morphism, $\delta: S \rightarrow S \times S$, followed by the multiplication, $\mu: S \times S \rightarrow S$. Thus, we have

$$
T_{e}(\mathrm{sq})=T_{(e, e)}(\mu) \circ T_{e}(\delta)
$$

with an obvious notation. Furthermore, $T_{e}(\delta): T_{e}(S) \rightarrow T_{e}(S) \times T_{e}(S)$ is the diagonal embedding; also, $T_{(e, e)}(\mu): T_{e}(S) \times T_{e}(S) \rightarrow T_{e}(S)$ equals $f_{\ell} \times f_{r}$, since the restriction of $\mu$ to $\{e\} \times S($ resp. $S \times\{e\})$ is just $e_{\ell}\left(\operatorname{resp} . e_{r}\right)$. Thus, $T_{e}(\mathrm{sq})=f_{\ell}+f_{r}$; this proves the claim.

Now (2) follows readily from the claim together with the decomposition (1). For the second assertion, let $z \in T_{e}(S)$ and write $z=z_{0,0}+z_{1,0}+z_{0,1}+z_{1,1}$ in that decomposition. Then $\left(f_{r}-f_{\ell}\right)(z)=z_{0,1}-z_{1,0}$ and hence $\operatorname{Im}\left(f_{r}-f_{\ell}\right)=T_{e}(S)_{1,0} \oplus T_{e}(S)_{0,1}$. 
Next, we observe that each joint eigenspace of $f_{\ell}$ and $f_{r}$ in $T_{e}(S)$ is the Zariski tangent space to a naturally defined closed subsemigroup scheme of $S$. Consider indeed the closed subscheme

$$
{ }_{e} S_{e}:=\{x \in S \mid e x=x e=e\},
$$

where the right-hand side is understood as the scheme-theoretic fiber at $e$ of the morphism $e_{\ell} \times e_{r}: S \rightarrow S \times S$. Define similarly

$$
e S_{e}:=\{x \in S \mid e x=x, x e=e\}, \quad{ }_{e} S e:=\{x \in S \mid e x=e, x e=x\},
$$

and finally

$$
e S e:=\{x \in S \mid e x=x e=x\}
$$

Then one readily obtains:

Lemma 2.6. With the above notation, ${ }_{e} S_{e}, e S_{e}, e_{e} S e$, and $e S e$ are closed subsemigroup schemes of $S$ containing e. Moreover, we have

$$
T_{e}\left({ }_{e} S_{e}\right)=T_{e}(S)_{0,0}, \quad T_{e}\left(e S_{e}\right)=T_{e}(S)_{1,0}, \quad T_{e}\left({ }_{e} S e\right)=T_{e}(S)_{0,1}, \quad T_{e}(e S e)=T_{e}(S)_{1,1} .
$$

Remarks 2.7. (i) Note that ${ }_{e} S_{e}$ is the largest closed subsemigroup scheme of $S$ containing $e$ as its zero. This subscheme is not necessarily reduced, as shown e.g. by [Br12, Ex. 3]. Specifically, consider the affine space $\mathbb{A}^{3}$ equipped with pointwise multiplication; this is a toric monoid. Let $M$ be the hypersurface of $\mathbb{A}^{3}$ with equation

$$
z^{n}-x y^{n}=0
$$

where $n$ is a positive integer. Then $M$ is a closed toric submonoid, containing $e:=(1,0,0)$ as an idempotent. Moreover, ${ }_{e} M_{e}$ is the closed subscheme of $\mathbb{A}^{3}$ with ideal generated by $x-1$ and $z^{n}-y^{n}$. Thus, ${ }_{e} M_{e}$ is everywhere nonreduced whenever $n$ is a multiple of the characteristic of $k$ (assumed to be nonzero).

(ii) Also, note that $e S_{e}$ is the largest closed subsemigroup scheme of $S$ containing $e$ and such that the composition law is the second projection. (Indeed, every such subsemigroup scheme $S^{\prime}$ satisfies $e x=x$ and $x e=e$ for any $T$-valued point $x$ of $S^{\prime}$, where $T$ is an arbitrary scheme; in other words, $S^{\prime} \subset e S_{e}$. Conversely, for any $T$-valued points $x, y$ of $e S_{e}$, we have $\left.x y=x e y=e y=y\right)$. In particular, $e S_{e}$ consists of idempotents, and $e S_{e}=x S_{x}$ for any $k$-rational point $x$ of $e S_{e}$.

Likewise, ${ }_{e} S e$ is the largest closed subsemigroup scheme of $S$ containing $e$ and such that the composition law is the first projection. We shall see in Corollary 2.9 that ${ }_{e} S e$ and $e S_{e}$ are in fact reduced.

(iii) Finally, $e S e$ is the largest closed submonoid scheme of $S$ with neutral element $e$. This subscheme is reduced, since it is the image of the morphism $S \rightarrow S, x \mapsto e x e$. Likewise, $S e$ and $e S$ are closed subsemigroups of $S$, and

$$
T_{e}(S e)=T_{e}(S)_{0,1} \oplus T_{e}(S)_{1,1}, \quad T_{e}(e S)=T_{e}(S)_{1,0} \oplus T_{e}(S)_{1,1}
$$


One would like to have a 'global' analogue of the decomposition (11) along the lines of the local structure results for algebraic monoids obtained in [Br08] (which makes an essential use of the unit group). Specifically, one would like to describe some open neighborhood of $e$ in $S$ by means of the product of the four pieces ${ }_{e} S_{e},{ }_{e} S e, e S_{e}$, and $e S e$ (taken in a suitable order) and of the composition law of $S$. But this already fails when $S$ is commutative: then both $e_{e} S e$ and $e S_{e}$ consist of the reduced point $e$, so that we only have two nontrivial pieces, $S_{e}$ and $e S$; moreover, the restriction of the composition law to $S_{e} \times e S \rightarrow S$ is just the second projection, since $x y=x e y=e y=y$ for all $x \in S_{e}$ and $y \in e S$. Yet we shall obtain global analogues of certain partial sums in the decomposition (1). For this, we introduce some notation.

Consider the algebraic monoid $e S e$ and its unit group, $G(e S e)$. Since $G(e S e)$ is open in $e S e$, the set

$$
U=U(e):=\{x \in S \mid \text { exe } \in G(e S e)\}
$$

is open in $S$. Clearly, $U$ contains $e$ and is stable under $e_{\ell}$ and $e_{r}$; also, note that

$$
U e \cap e U=e U e=G(e S e)
$$

We now describe the structure of $U e$ :

Lemma 2.8. Keep the above notation.

(i) $U e=\{x \in S e \mid e x \in G(e S e)\}$ and $e_{e} U e={ }_{e} S e$.

(ii) $U$ e is an open subsemigroup of $S e$.

(iii) The morphism

$$
\varphi:{ }_{e} S e \times G(e S e) \longrightarrow S, \quad(x, g) \longmapsto x g
$$

is a locally closed immersion with image Ue. Moreover, $\varphi$ is an isomorphism of semigroup schemes, where the composition law of the left-hand side is given by $(x, g)(y, h):=(x, g h)$. (iv) The tangent map of $\varphi$ at $(e, e)$ induces an isomorphism

$$
T_{e}(S)_{0,1} \oplus T_{e}(S)_{1,1} \cong T_{e}(U e)=T_{e}(S e)
$$

Proof. Both assertions of (i) are readily checked. The first assertion implies that $U e$ is open in $S e$. To show that $U e$ is a subsemigroup, note that for any points $x, y$ of $U e$, we have $e x y=e x e y$. Hence $e x y \in G(e S e)$ by (i), so that $x y \in U e$ by (i) again. This completes the proof of (ii).

For (iii), consider $T$-valued points $x$ of $e_{e} S e$ and $g$ of $G(e S e)$, where $T$ is an arbitrary scheme. Then exge $=e g e=g$ and hence $x g$ is a $T$-valued point of $U e$. Thus, $\varphi$ yields a $\operatorname{morphism}_{e} S e \times G(e S e) \rightarrow U e$. Moreover, we have

$$
\varphi(x, g) \varphi(y, h)=x g y h=x g e y h=x g e h=x g h=\varphi((x, g)(y, h)),
$$

that is, $\varphi$ is a homomorphism of semigroup schemes. To show that $\varphi$ is an isomorphism, consider a $T$-valued point $z$ of $U e$ and denote by $(e z)^{-1}$ the inverse of $e z$ in $G(e S e)$. Then $z=x g$, where $x:=z(e z)^{-1}$ and $g:=e z$; moreover, $x \in\left({ }_{e} S e\right)(T)$ and $g \in(e S e)(T)$. Also, if $z=y h$ where $y \in\left({ }_{e} S e\right)(T)$ and $h \in(e S e)(T)$, then $h=e h=e y h=e z$ and $y=y e=y h h^{-1}=z(e z)^{-1}$. Thus, the morphism

$$
U e \longrightarrow e_{e} S e \times G(e S e), \quad z \longmapsto\left(z(e z)^{-1}, e z\right)
$$


is the inverse of $\varphi$.

Finally, (iv) follows readily from (iii) in view of Lemma 2.6.

Corollary 2.9. (i) The scheme ${ }_{e} S e$ is reduced, and is a union of connected components of $E(S e)$.

(ii) If $S$ is irreducible, then ${ }_{e} S e$ is the unique irreducible component of $E(S e)$ through e.

Proof. Since $U e$ is reduced, and isomorphic to ${ }_{e} S e \times G(e S e)$ in view of Lemma 2.8, we see that ${ }_{e} S e$ is reduced as well. Moreover, that lemma also implies that ${ }_{e} S e=E(S e) \cap U e$ (as schemes). In particular, ${ }_{e} S e$ is open in $E(S e)$. But ${ }_{e} S e$ is also closed; this proves (i).

Next, assume that $S$ is irreducible; then so are $S e$ and $U e$. By Lemma 2.8 again, ${ }_{e} S e$ is irreducible as well, which implies (ii).

Note that a dual version of Lemma 2.8 yields the structure of $e U$; also, $e S_{e}$ satisfies the dual statement of Corollary 2.9.

We now obtain a description of the isolated points of $E(S)$ (viewed as a topological space). To state our result, denote by $C=C(e)$ the union of those irreducible components of $S$ that contain $e$, or alternatively, the closure of any neighborhood of $e$ in $S$; then $C$ is a closed subsemigroup of $S$.

Proposition 2.10. With the above notation, $e$ is isolated in $E(S)$ if and only if $e$ centralizes $C$; then $E(S)$ is reduced at e.

In particular, the isolated idempotents of an irreducible algebraic semigroup are exactly the central idempotents.

Proof. Assume that $e$ centralizes $C$; then $e_{\ell}$ and $e_{r}$ induce the same endomorphism of the local ring $\mathcal{O}_{C, e}=\mathcal{O}_{S, e}$. Thus, $f_{\ell}=f_{r}$. By Lemma 2.5, it follows that $T_{e}(E(S))=\{0\}$. Hence $e$ is an isolated reduced point of $E(S)$.

Conversely, if $e$ is isolated in $E(S)$, then it is also isolated in ${ }_{e} S e$ and in $e S_{e}$ (since they both consist of idempotents). As $e S e$ and $e S_{e}$ are reduced, it follows that $T_{e}\left(e_{e} S e\right)=$ $\{0\}=T_{e}\left(e S_{e}\right)$, i.e., $T_{e}(S)_{0,1}=T_{e}(S)_{1,0}=\{0\}$. In view of Lemma 2.5, we thus have $T_{e}(E(S))=\{0\}$, i.e., $E(S)$ is reduced at $e$. Moreover, $f_{\ell}=f_{r}$ by Lemma 2.5 again. In other words, $e_{\ell}$ and $e_{r}$ induce the same endomorphism of $\mathfrak{m} / \mathfrak{m}^{2}$, where $\mathfrak{m}$ denotes the maximal ideal of $\mathcal{O}_{S, e}$. Hence $e_{\ell}$ and $e_{r}$ induce the same endomorphism of $\mathfrak{m}^{n} / \mathfrak{m}^{n+1}$ for any integer $n \geq 1$, since the natural map $\operatorname{Sym}^{n}\left(\mathfrak{m} / \mathfrak{m}^{2}\right) \rightarrow \mathfrak{m}^{n} / \mathfrak{m}^{n+1}$ is surjective and equivariant for the natural actions of $e_{\ell}$ and $e_{r}$. Next, consider the endomorphisms of $\mathcal{O}_{S, e} / \mathfrak{m}^{n}$ induced by $e_{r}$ and $e_{\ell}$ : these are commuting idempotents of this finite-dimensional $k$-vector space, which preserve the filtration by the subspaces $\mathfrak{m}^{m} / \mathfrak{m}^{n}(0 \leq m \leq n)$ and coincide on the associated graded vector space. Thus, $e_{\ell}=e_{r}$ as endomorphisms of $\mathcal{O}_{S, e} / \mathfrak{m}^{n}$ for all $n$, and hence as endomorphisms of $\mathcal{O}_{S, e}$. This means that for any $f \in \mathcal{O}_{S, e}$ there exists a neighborhood $V=V_{f}$ of $e$ in $S$ such that $f(e x)=f(x e)$ for all $x \in V$. Since $\mathcal{O}_{S, e}$ is the localization of a finitely generated $k$-algebra, it follows that we may choose $V$ independently of $f$. Then $x e=e x$ for all $x \in V$, and hence for all $x \in C$, since $V$ is dense in $C$.

We now return to the decomposition (11), and obtain a global analogue of the partial sum $T_{e}(S)_{1,0} \oplus T_{e}(S)_{0,1} \oplus T_{e}(S)_{1,1}$ in terms of the open subset $U$ : 
Lemma 2.11. (i) The morphism

$$
\psi:{ }_{e} S e \times G(e S e) \times e S_{e} \longrightarrow S, \quad(x, g, y) \longmapsto x g y
$$

is a locally closed immersion with image $U e U$.

(ii) The tangent map of $\psi$ at $(e, e, e)$ induces an isomorphism

$$
T_{e}(S)_{0,1} \oplus T_{e}(S)_{1,1} \oplus T_{e}(S)_{1,0} \cong T_{e}(U e U)
$$

Proof. (i) Let $z \in U e U$; then $z=z^{\prime} z^{\prime \prime}$ with $z^{\prime} \in U e$ and $z^{\prime \prime} \in e U$. In view of Lemma 2.8, it follows that $z=x g y$ with $x \in{ }_{e} S e, g \in G(e S e)$, and $y \in e S_{e}$. Then $z e=$ $x g e=x g$; likewise, $e z=e g y=g y$. Thus, we have $g=e z e, x=z e(e z e)^{-1}$, and $y=(e z e)^{-1} e z$. In particular, $z$ satisfies the following conditions: $z \in U$, and $z=$ $z e(e z e)^{-1}(e z e)(e z e)^{-1} e z$. Conversely, if $z \in S$ satisfies the above two conditions, then $z \in_{e} S e G(e S e) e S_{e} \subset(U e)(e U)=U e U$. Also, these conditions clearly define a locally closed subset of $S$. This yields the assertions.

(ii) follows from (i) in view of Lemma 2.6.

Finally, we obtain a parameterization of those idempotents of $S$ that are contained in $U e U$. To state it, let

$$
V=V(e):=\left\{(x, y) \in{ }_{e} S e \times e S_{e} \mid y x \in G(e S e)\right\}
$$

Then $V$ is an open neighborhood of $(e, e)$ in ${ }_{e} S e \times e S_{e}$. For any point $(x, y)$ of $V$, we denote by $(y x)^{-1}$ the inverse of $y x$ in $G(e S e)$.

Lemma 2.12. With the above notation, the morphism

$$
\gamma: V \longrightarrow S, \quad(x, y) \longmapsto x(y x)^{-1} y
$$

induces an isomorphism from $V$ to the scheme-theoretic intersection $U e U \cap E(S)$. Moreover, the tangent map of $\gamma$ at $(e, e)$ induces an isomorphism

$$
T_{0,1}(S) \oplus T_{1,0}(S) \cong T_{e}(U e U \cap E(S)) .
$$

Proof. We argue with $T$-valued points for an arbitrary scheme $T$, as in the proof of Lemma 2.8 (iii).

Let $z \in U e U$. By Lemma 2.11, we may write $z$ uniquely as $x g y$, where $x \in{ }_{e} S e$, $g \in G(e S e)$, and $y \in e S_{e}$. If $z \in E(S)$, then of course xgyxgy =xgy. Multiplying by $e$ on the left and right, this yields $g y x g=g$ and hence $g y x=e$. Thus, $(x, y) \in V$ and $z=\gamma(x, y)$. Conversely, if $(x, y) \in V$, then $x(y x)^{-1} \in{ }_{e} S e G(e S e)$ and hence $x(y x)^{-1} \in U e$ by Lemma 2.8. Using that lemma again, it follows that $\gamma(x, y) \in U e U$. Also, one readily checks that $\gamma(x, y)$ is idempotent. This shows the first assertion, which in turn implies the second assertion.

Remarks 2.13. (i) The above subsets $U e, e U, U e U$, and $e U e$ are contained in the corresponding equivalence classes of $e$ under Green's relations (see e.g. [Pu88, Def. 1.1] for the definition of these relations). 
Indeed, for any $x \in U e$, we have obviously $S^{1} x \subset S^{1} e=S e$, where $S^{1}$ denotes the monoid obtained from $S$ by adjoining a neutral element. Also, $S^{1} x$ contains $(e x)^{-1} e x=e$. Thus, $S^{1} x=S^{1} e$, that is, $x \mathcal{L} e$ with the notation of [loc. cit.]. Likewise, $x \mathcal{R} e$ for any $x \in e U$, and $x \mathcal{J} e$ for any $x \in U e U$. Finally, $e U e=G(e S e)$ equals the $\mathcal{H}$-equivalence class of $e$.

Also, one readily checks that $e S_{e}$ (resp. $e_{e} S e$ ) is the set of idempotents in the equivalence class of $e$ under $\mathcal{R}$ (resp. $\mathcal{L}$ ).

(ii) Consider the centralizer of $e$ in $S$,

$$
C_{S}(e):=\{x \in S \mid x e=e x\} .
$$

This is a closed subsemigroup scheme of $S$ containing both $e S e$ and ${ }_{e} S_{e}$. Moreover, the (left or right) multiplication by $e$ yields a retraction of semigroup schemes $C_{S}(e) \rightarrow e S e$, and we have

$$
T_{e} C_{S}(e)=T_{e}(S)_{0,0} \oplus T_{e}(S)_{1,1} .
$$

We may also consider the left centralizer of $e$ in $S$,

$$
C_{S}^{\ell}(e):=\{x \in S \mid e x=e x e\} .
$$

This is again a closed subsemigroup scheme of $S$, which contains both $S e$ and $e_{e} S_{e}$. Also, one readily checks that the left multiplication $e_{\ell}$ yields a retraction of semigroup schemes $C_{S}^{\ell}(e) \rightarrow e S e$, and we have

$$
T_{e} C_{S}^{\ell}(e)=T_{e}(S)_{0,0} \oplus T_{e}(S)_{0,1} \oplus T_{e}(S)_{1,1} .
$$

Moreover, $C_{S}^{\ell}(e) \cap U$ is the preimage of $G(e S e)$ under $e_{\ell}$.

The right centralizer of $e$ in $S$,

$$
C_{S}^{r}(e):=\{x \in S \mid x e=e x e\}
$$

satisfies similar properties; note that $C_{S}(e)=C_{S}^{\ell}\left(C_{S}^{r}(e)\right)=C_{S}^{r}\left(C_{S}^{\ell}(e)\right)$. Also, one easily checks that $U$ is stable under $C_{S}^{\ell}(e) \times C_{S}^{r}(e)$ acting on $S$ by left and right multiplication. (iii) Recall the description of Green's relations for an algebraic monoid $M$ with dense unit group $G$ (see [Pu84, Thm. 1]). For any $x, y \in M$, we have:

$$
\begin{gathered}
x \mathcal{L} y \Leftrightarrow \overline{M x}=\overline{M y} \Leftrightarrow G x=G y, \quad x \mathcal{R} y \Leftrightarrow \overline{x M}=\overline{y M} \Leftrightarrow x G=y G, \\
x \mathcal{J} y \Leftrightarrow \overline{M x M}=\overline{M y M} \Leftrightarrow G x G=G y G .
\end{gathered}
$$

(Indeed, $x \mathcal{L} y$ if and only if $M x=M y$; then $\overline{M x}=\overline{M y}$. Since $G x$ is the unique dense open $G$-orbit in $\overline{M x}$, it follows that $G x=G y$. Conversely, if $G x=G y$ then $M x=M y$. This proves the first equivalence; the next ones are checked similarly).

In view of (i), it follows that $U e \subset G e$ for any idempotent $e$ of $M$. Likewise, $e U \subset e G$ and hence $U e U \subset G e G$; also, $e U e \subset e G e$. These inclusions are generally strict, e.g., when $M$ is the monoid of $n \times n$ matrices and $e \neq 0,1$. Thus, $U e$ is in general strictly contained in the $\mathcal{L}$-class of $e$, and likewise for $e U, U e U$.

Also, in view of (ii), $U$ is stable under left multiplication by $C_{G}^{\ell}(e)$ and right multiplication by $C_{G}^{r}(e)$. In particular, $U e$ is an open subset of $M e$ containing $C_{G}^{\ell}(e) e$. If $M$ is irreducible, then $C_{G}^{\ell}(e) e$ is open in $M e$ by $[\mathrm{Pu} 88$, Thm. 6.16 (ii)]. As a consequence, $U$ contains the open $C_{G}^{\ell}(e) \times C_{G}^{r}(e)$-stable neighborhood $M_{0}$ of $e$ in $M$, whose structure is described in [Br08, Thm. 2.2.1]. Yet $M_{0}$ is in general strictly contained in $U$. 


\subsection{Smoothness}

In this subsection, we first obtain a slight generalization of Theorem 1.1, we then apply the result to intervals in idempotents of irreducible algebraic semigroups.

Recall that an algebraic monoid $M$ is unit dense if it is the closure of its unit group; this holds e.g. when $M$ is irreducible. We may now state:

Theorem 2.14. Let $M$ be a unit dense algebraic monoid, $G$ its unit group, and $T$ a maximal torus of $G$; denote by $M^{\circ}$ (resp. $G^{o}$ ) the neutral component of $M$ (resp. of $G$ ), and by $\bar{T}$ the closure of $T$ in $M$. Then the scheme $E(M)$ is smooth, and equals $E\left(M^{\circ}\right)$. Moreover, the connected components of $E(M)$ are conjugacy classes of $G^{o}$; every such component meets $\bar{T}$.

Proof. Consider an idempotent $e \in M$, and its open neighborhood $U$ defined by (3). Then $U e U$ is a locally closed subvariety of $M$ by Lemma 2.11. We claim that $U e U$ is smooth at $e$.

To prove the claim, note that the subset $G e G$ of $M$ is a smooth, locally closed subvariety, since it is an orbit of the algebraic group $G \times G$ acting on $M$ by left and right multiplication. Also, $G e G \supset U e U \supset(G \cap U) e(G \cap U)$, where the first inclusion follows from Remark 2.13 (iii). Moreover, $G \cap U$ is an open neighborhood of $e$, dense in $U$ (as $G$ is dense in $M)$. Since the orbit map $G \times G \rightarrow G e G,(x, y) \mapsto x e y$ is flat, it follows that $(G \cap U) e(G \cap U)$ is an open neighborhood of $e$ in $G e G$, and hence in $U e U$. This yields the claim.

By that claim together with Lemma 2.11, the schemes ${ }_{e} M e$ and $e M_{e}$ are smooth at $e$. In view of Lemma 2.12, it follows that $e$ is contained in a smooth, locally closed subvariety $V$ of $E(M)$ such that

$$
\operatorname{dim}_{e}(V)=\operatorname{dim}_{e}(e M e)+\operatorname{dim}_{e}\left(e M_{e}\right) .
$$

Using Lemmas 2.5 and 2.6, we obtain

$$
\operatorname{dim}_{e}(V)=\operatorname{dim} T_{e}(M)_{0,1}+\operatorname{dim} T_{e}(M)_{1,0}=\operatorname{dim} T_{e}(E(M)) .
$$

Thus, $V$ contains an open neighborhood of $e$ in $E(M)$; in particular, $E(M)$ is smooth at $e$. We have shown that the scheme $E(M)$ is smooth.

Next, recall that $M^{o}$ is a closed irreducible submonoid of $M$ with unit group $G^{o}$ (see [Br12, Prop. 2, Prop. 6]). Also, $E(M)=E\left(M^{o}\right)$ as sets, in view of [loc. cit., Rem. 6 (ii)]. Since $E(M)$ is smooth, it follows that $E(M)=E\left(M^{o}\right)$ as schemes.

To complete the proof, we may replace $M$ with $M^{o}$ and hence assume that $M$ is irreducible; then $G$ is connected. Thus, $G$ has a largest closed connected affine normal subgroup, $G_{\text {aff }}$ (see e.g. [Ro56, Thm. 16, p. 439]). Denote by $M_{\text {aff }}$ the closure of $G_{\text {aff }}$ in $M$. By [Br12, Thm. 3], $M_{\text {aff }}$ is an irreducible affine algebraic monoid with unit group $G_{\text {aff }}$, and $E(M)=E\left(M_{\text {aff }}\right)$ as sets. Thus, we may further assume that $M$ is affine, or equivalently linear (see [Pu88, Thm. 3.15]). Then every conjugacy class in $E(M)$ meets $\bar{T}$ by [loc. cit., Cor. 6.10]. Moreover, $E(\bar{T})$ is finite by [loc. cit., Thm. 8.4] (or alternatively by Proposition 2.3). Thus, it suffices to check that the $G$-conjugacy class of every $e \in E(\bar{T})$ is closed in $M$.

This assertion is shown in [Br08, Lem. 1.2.3] under the additional assumption that $k$ has characteristic 0 . Yet that assumption is unnecessary; we recall the argument for the 
convenience of the reader. Let $B$ be a Borel subgroup of $G$ containing $T$; since $G / B$ is complete, it suffices to show that the $B$-conjugacy class of $e$ is closed in $M$. But that class is also the $U$-conjugacy class of $e$, where $U$ denotes the unipotent part of $B$; indeed, we have $B=U T$, and $T$ centralizes $e$. So the desired closedness assertion follows from the fact that all orbits of a unipotent algebraic group acting on an affine variety are closed (see e.g. [Sp98, Prop. 2.4.14]).

Remarks 2.15. (i) If $S$ is a smooth algebraic semigroup, then the scheme $E(S)$ is smooth as well. Indeed, for any idempotent $e$ of $S$, the variety $S e$ is smooth (since it is the image of the smooth variety $S$ under the retraction $e_{r}$ ). In view of Lemma 2.8, it follows that ${ }_{e} S e$, and likewise $e S_{e}$, are smooth at $e$. This implies in turn that $E(S)$ is smooth at $e$, by arguing as in the third paragraph of the proof of Theorem 2.14,

(ii) In particular, the scheme of idempotents of any finite-dimensional associative algebra $A$ is smooth. This can be proved directly as follows. Firstly, one reduces to the case of an unital algebra: consider indeed the algebra $B:=k \times A$, where the multiplication is given by $(t, x)(u, y):=(t u, t y+u x+x y)$. Then $B$ is a finite-dimensional associative algebra with unit $(1,0)$. Moreover, one checks that the scheme $E(B)$ is the disjoint union of two copies of $E(A)$ : the images of the morphisms $x \mapsto(0, x)$ and $x \mapsto(1,-x)$. Secondly, if $A$ is unital with unit group $G$, and $e \in A$ is idempotent, then the tangent map at 1 of the orbit map

$$
G \longrightarrow A, \quad g \longmapsto g e g^{-1}
$$

is identified with $f_{r}-f_{\ell}$ under the natural identifications of $T_{1}(G)$ and $T_{e}(A)$ with $A$. In view of Lemma 2.5, it follows that the conjugacy class of $e$ contains a neighborhood of $e$ in $E(A)$; this yields the desired smoothness assertion.

(iii) One may ask for a simpler proof of Theorem 2.14 based on a tangent map argument as above. But in the setting of that theorem, there seems to be no relation between the Zariski tangent spaces of $M$ at the smooth point $1_{M}$ and at the (generally singular) point $e$.

Still considering a unit dense algebraic monoid $M$ with unit group $G$, we now describe the isotropy group scheme of any idempotent $e \in M$ for the $G$-action by conjugation, i.e., the centralizer $C_{G}(e)$ of $e$ in $G$. Recall from Remark 2.13 (ii) that the centralizer of $e$ in $M$ is equipped with a retraction of monoid schemes $\tau: C_{M}(e) \rightarrow e M e$; thus, $\tau$ restricts to a retraction of group schemes that we still denote by $\tau: C_{G}(e) \rightarrow G(e M e)$. We may now state the following result, which generalizes [Br08, Lem. 1.2.2 (iii)] with a more direct proof:

Proposition 2.16. With the above notation, we have an exact sequence of group schemes

$$
1 \longrightarrow{ }_{e} G_{e} \longrightarrow C_{G}(e) \stackrel{\tau}{\longrightarrow} G(e M e) \longrightarrow 1 \text {. }
$$

Proof. Clearly, the scheme-theoretic kernel of $\tau: C_{G}(e) \rightarrow G(e M e)$ equals ${ }_{e} G_{e}$. Since $G(e M e)$ is reduced, it remains to show that $\tau$ is surjective on $k$-rational points. For this, consider the left stabilizer $C_{M}^{\ell}(e)$ equipped with its reduced subscheme structure. This is a closed submonoid of $M$; moreover, the map

$$
\tau^{\ell}: C_{M}^{\ell}(e) \longrightarrow e M e, \quad x \longmapsto e x
$$


is a retraction and a homomorphism of algebraic monoids (see Remark 2.13 (ii) again). Also, $C_{G}^{\ell}(e):=C_{M}^{\ell}(e) \cap G$ is a closed subsemigroup of $G$, and hence a closed subgroup by [Re05, Exc. 3.5.1.2]. This yields a homomorphism of algebraic groups that we still denote by $\tau^{\ell}: C_{G}^{\ell}(e) \rightarrow G(e M e)$.

We claim that the latter homomorphism is surjective. Indeed, let $x \in G(e M e)$. Then $x M=e M$, since $x \in e M$ and $e=x x^{-1} \in x M$. As $x G$ is the unique dense $G$-orbit in $x M$ for the $G$-action on $M$ by right multiplication, it follows that $x G=e G$. Hence there exists $g \in G$ such that $x=e g$; then $e g e=x e=x=e g$, i.e., $x \in C_{G}^{\ell}(e)$. This proves the claim.

Next, observe that $\overline{C_{G}^{\ell}(e)}$ (closure in $M$ ) is a unit dense submonoid of $M$. Moreover, with the notation of Theorem 2.14, we have $e \in \bar{T}$ and hence $T \subset C_{G}(e) \subset C_{G}^{\ell}(e)$; thus, $e \in \overline{C_{G}^{\ell}(e)}$. Therefore, $G(e M e)=e C_{G}^{\ell}(e)$ is contained in $\overline{C_{G}^{\ell}(e)}$ as well. Thus, to show the desired surjectivity, we may replace $M$ with $\overline{C_{G}^{\ell}(e)}$. Then we apply the claim to the right stabilizer $C_{G}^{r}(e)$; this yields the statement, since $C_{G}^{r}\left(C_{G}^{\ell}(e)\right)=C_{G}(e)$.

Finally, we apply Theorem 2.14 to the structure of intervals in $E(S)$, where $S$ is an algebraic semigroup. Given two idempotents $e_{0}, e_{1} \in S$ such that $e_{0} \leq e_{1}$, we consider

$$
\left[e_{0}, e_{1}\right]:=\left\{x \in E(S) \mid e_{0} \leq x \leq e_{1}\right\} .
$$

This has a natural structure of closed subscheme of $S$, namely, the scheme-theoretic intersection $E(S) \cap e_{e_{0}} S_{e_{0}} \cap e_{1} S e_{1}$ (since $e_{0} \leq x$ if and only if $x \in e_{e_{0}} S_{e_{0}}$, and $x \leq e_{1}$ if and only if $\left.x \in e_{1} S e_{1}\right)$. Note that $e_{1} S e_{1}$ is a closed submonoid of $S$ containing $e_{0}$. Moreover,

$$
{ }_{e_{0}} S_{e_{0}} \cap e_{1} S e_{1}={ }_{e_{0}}\left(e_{1} S e_{1}\right)_{e_{0}}=: M\left(e_{0}, e_{1}\right)=M
$$

is a closed submonoid scheme of $e_{1} S e_{1}$ with zero $e_{0}$, and $\left[e_{0}, e_{1}\right]=E(M)$ as schemes. We may now state the following result, which sharpens and builds on a result of Putcha (see [Pu88, Thm. 6.7]):

Corollary 2.17. Keep the above notation, and assume that $k$ has characteristic 0 and $S$ is irreducible. Then $M$ is reduced, affine, and unit dense. Moreover, the interval $\left[e_{0}, e_{1}\right]$ is smooth; each connected component of $\left[e_{0}, e_{1}\right]$ is a conjugacy class of $G(M)^{\circ}$.

Proof. We may replace $S$ with $e_{1} S e_{1}$; thus, we may assume that $S$ is an irreducible algebraic monoid, and $e_{1}=1_{S}$. Then $M={ }_{e_{0}} S_{e_{0}}$ is reduced, as follows from the local structure of $S$ at $e_{0}$ (see [Br08]); more specifically, an open neighborhood of $e_{0}$ in $M$ is isomorphic to a homogeneous fiber bundle with fiber ${ }_{e_{0}} M_{e_{0}}$ by [loc. cit., Thm. 2.2.1, Rem. 3.1.3]. Moreover, $M$ is affine and unit dense by [loc. cit., Lem. 3.1.4]. The final assertion follows from these results in view of Theorem 2.14 .

Note that the above statement does not extend to positive characteristics. Indeed, $M$ can be nonreduced in that case, as shown by the example in Remark 2.7 (i). 


\section{Irreducible commutative algebraic semigroups}

\subsection{The finite poset of idempotents}

Throughout this subsection, we consider an irreducible commutative algebraic semigroup $S$. We first record the following easy result:

Proposition 3.1. $S$ has a largest idempotent, $e_{1}$. Moreover, there exists a positive integer $n$ such that $x^{n} \in e_{1} S$ for all $x \in S$. If $S$ is defined over $F$, then so is $e_{1}$.

Proof. By the finiteness of $E(S)$ (Proposition 2.3) together with [BrRe12, Cor. 1], there exist a positive integer $n$, an idempotent $e_{1} \in S$, and a nonempty open subset $U$ of $S$ such that $x^{n}$ is a unit of the closed submonoid $e_{1} S$ for all $x \in U$. Since $S$ is irreducible, it follows that $x^{n} \in e_{1} S$ for all $x \in S$. In particular, $e \in e_{1} S$ for any $e \in E(S)$, i.e., $e=e_{1} e$; hence $e_{1}$ is the largest idempotent.

If $S$ is defined over $F$, then $e_{1}$ is defined over $F_{s}$ (by Proposition 2.3 again) and is clearly invariant under $\Gamma$. Thus, $e_{1} \in E(S)(F)$.

With the above notation, the unit group $G\left(e_{1} S\right)$ is a connected commutative algebraic group, and hence has a largest subtorus, $T$. The closure $\bar{T}$ of $T$ in $S$ is a closed toric submonoid with neutral element $e_{1}$ and unit group $T$. We now gather further properties of $\bar{T}$ :

Proposition 3.2. With the above notation, we have:

(i) $E(S)=E(\bar{T})$.

(ii) $\bar{T}$ contains every subtorus of $S$.

(iii) If $S$ is defined over $F$, then so is $\bar{T}$.

Proof. (i) Note that $\bar{T}=e_{1} \bar{T}$, and $E(S)=e_{1} E(S)=E\left(e_{1} S\right)$. Thus, we may replace $S$ with $e_{1} S$, and hence assume that $S$ is an irreducible commutative algebraic monoid. Then the first assertion follows from Theorem 2.14 .

(ii) Let $S^{\prime}$ be a subtorus of $S$ (i.e., a locally closed subsemigroup which is isomorphic to a torus as an algebraic semigroup), and denote by $e$ the neutral element of $S^{\prime}$. Then $S^{\prime}=e S^{\prime} \subset e S=e_{1} e S \subset e_{1} S$. Hence we may again replace $S$ with $e_{1} S$, and assume that $S$ is an irreducible commutative algebraic monoid. Now consider the map

$$
\varphi: S \longrightarrow e S, \quad x \longmapsto x e .
$$

Then $\varphi$ is a surjective homomorphism of algebraic monoids. Thus, $\varphi$ restricts to a homomorphism of unit groups $G:=G(S) \rightarrow G(e S)$; the image of that homomorphism is closed, and also dense since $G$ is dense in $S$. Thus, $\varphi$ sends $G$ onto $G(e S)$. Since $S^{\prime}=G\left(e \overline{S^{\prime}}\right)$ is a closed connected subgroup of $G(e S)$, there exists a closed connected subgroup $G^{\prime}$ of $G$ such that $\varphi\left(G^{\prime}\right)=S^{\prime}$. Let $G_{\text {aff }}^{\prime}$ denote the largest closed connected affine subgroup of $G^{\prime}$. Since $S^{\prime}$ is affine, we also have $\varphi\left(G_{\text {aff }}^{\prime}\right)=S^{\prime}$, as follows from the decomposition $G^{\prime}=G_{\text {aff }}^{\prime} G_{\text {ant }}^{\prime}$, where $G_{\text {ant }}^{\prime}$ denotes the largest closed subgroup of $G$ such that every homomorphism from $G_{\text {ant }}^{\prime}$ to an affine algebraic group is constant (see e.g. [Ro56, Cor. 5, pp. 440-441]). But in view of the structure of commutative affine algebraic groups (see e.g. [Sp98, Thm. 3.1.1]), we have $G_{\text {aff }}^{\prime}=T^{\prime} \times U^{\prime}$, where $T^{\prime}$ (resp. $U^{\prime}$ ) denotes the largest subtorus (resp. the largest 
unipotent subgroup) of $G_{\text {aff }}^{\prime}$. Thus, $\varphi\left(T^{\prime}\right)=S^{\prime}$, that is, $S^{\prime}=e T^{\prime}$. Since $T^{\prime} \subset T$ and $e \in \bar{T}$, it follows that $S^{\prime} \subset \bar{T}$.

(iii) Since $e_{1}$ is defined over $F$, so is $e_{1} S$. Hence the algebraic group $G\left(e_{1} S\right)$ is also defined over $F$, by [Sp98, Prop. 11.2.8 (ii)]. In view of [SGA3, Exp. XIV, Thm. 1.1], it follows that the largest subtorus $T$ of $G\left(e_{1} S\right)$ is defined over $F$ as well. Thus, so is $\bar{T}$.

\subsection{Toric monoids}

As mentioned in the introduction, the toric monoids are exactly the affine toric varieties (not necessarily normal). For later use, we briefly discuss their structure; details can be found e.g. in [Ne92, [Pu81, §2, §3], and [Re05, §3.3].

The isomorphism classes of toric monoids are in a bijective correspondence with the pairs $(\Lambda, \mathcal{M})$, where $\Lambda$ is a free abelian group and $\mathcal{M}$ is a finitely generated submonoid of $\Lambda$ which generates that group. This correspondence assigns to a toric monoid $M$ with unit group $T$, the lattice $\Lambda$ of characters of $T$ and the monoid $\mathcal{M}$ of weights of $T$ acting on the coordinate ring $\mathcal{O}(M)$ via its action on $M$ by multiplication. Conversely, one assigns to a pair $(\Lambda, \mathcal{M})$, the torus $T:=\operatorname{Hom}\left(\Lambda, \mathbb{G}_{m}\right)$ (consisting of all group homomorphisms from $\Lambda$ to the multiplicative group) and the monoid $M:=\operatorname{Hom}\left(\mathcal{M}, \mathbb{A}^{1}\right)$ (consisting of all monoid homomorphisms from $\mathcal{M}$ to the affine line equipped with the multiplication). The coordinate ring $\mathcal{O}(T)$ (resp. $\mathcal{O}(M)$ ) is identified with the group ring $k[\Lambda]$ (resp. the monoid ring $k[\mathcal{M}])$.

Via this correspondence, the idempotents of $M$ are identified with the monoid homomorphisms $\varepsilon: \mathcal{M} \rightarrow\{1,0\}$. Any such homomorphism is uniquely determined by the preimage of 1 , which is the intersection of $\mathcal{M}$ with a unique face of the cone, $C(\mathcal{M})$, generated by $\mathcal{M}$ in the vector space $\Lambda \otimes_{\mathbb{Z}} \mathbb{R}$. Moreover, every $T$-orbit in $M$ (for the action by multiplication) contains a unique idempotent. This defines bijective correspondences between the idempotents of $M$, the faces of the rational, polyhedral, convex cone $C(\mathcal{M})$, and the $T$-orbits in $M$. Via these correspondences, the partial order relation on $E(M)$ is identified with the inclusion of faces, resp. of orbit closures; moreover, the dimension of a face is the dimension of the corresponding orbit. In particular, there is a unique closed orbit, corresponding to the minimal idempotent and to the smallest face of $C(\mathcal{M})$ (which is also the largest linear subspace contained in $C(\mathcal{M})$ ).

The toric monoid $M$ is defined over $F$ if and only if $\Lambda$ is equipped with a continuous action of $\Gamma$ that stabilizes $\mathcal{M}$ (this is shown e.g. in [Sp98, Prop. 3.2.6] for tori; the case of toric monoids is handled by similar arguments). Under that assumption, the above correspondences are compatible with the actions of $\Gamma$.

We also record the following observation:

Lemma 3.3. Let $M$ be a toric monoid, and $S$ a closed irreducible subsemigroup of $M$. Then $S$ is a toric monoid.

Proof. Since $S$ is irreducible, there is a unique $T$-orbit in $M$ that contains a dense subset of $S$. Thus, there exists a unique idempotent $e_{S}$ of $M$ such that $S \cap e_{S} T$ is dense in $S$. Then $S \cap e_{S} T$ is a closed irreducible subsemigroup of the torus $e_{S} T$, and hence a subtorus in view of [Re05, Exc. 3.5.1.2]. This yields our assertion. 
Next, consider a toric monoid $M$ with unit group $T$ and smallest idempotent $e_{0}$, so that the closed $T$-orbit in $M$ is $e_{0} T$. Denote by $T_{0}$ the reduced neutral component of the isotropy group $T_{e_{0}}$, and by $\overline{T_{0}}$ the closure of $T_{0}$ in $M$. Clearly, $\overline{T_{0}}$ is a toric monoid with torus $T_{0}$ and zero $e_{0}$; this monoid is the reduced scheme of the monoid scheme $M\left(e_{0}, e_{1}\right)$ defined by (4). We now gather further properties of $\overline{T_{0}}$ :

Proposition 3.4. With the above notation, we have:

(i) $\operatorname{dim}\left(T_{0}\right)$ equals the length of any maximal chain of idempotents in $M$.

(ii) $\overline{T_{0}}$ is the smallest closed irreducible subsemigroup of $M$ containing $E(M)$.

(iii) $\overline{T_{0}}$ is the largest closed irreducible subsemigroup of $M$ having a zero.

(iv) If $M$ is defined over $F$, then so is $\overline{T_{0}}$.

Proof. (i) Since $e_{0} T$ is closed in $M$, we easily obtain that $e_{0} M=e_{0} T$. Also, $e_{0} T$ is isomorphic to the homogeneous space $T / T_{e_{0}}$, where $T_{e_{0}}$ denotes the (scheme-theoretic) stabilizer of $e_{0}$ in $T$. Thus, the morphism $\varphi: M \rightarrow e_{0} M, x \mapsto e_{0} x$ makes $M$ a $T$ homogeneous fiber bundle over $T / T_{e_{0}}$; its scheme-theoretic fiber at $e_{0}$ is the closure of $T_{e_{0}}$ in $M$. Therefore, we have

$$
\operatorname{dim}(M)-\operatorname{dim}\left(e_{0} M\right)=\operatorname{dim}\left(T_{e_{0}}\right)=\operatorname{dim}\left(T_{0}\right) .
$$

Thus, $\operatorname{dim}\left(T_{0}\right)$ is the codimension of the smallest face of the cone associated with $M$. This also equals the length of any maximal chain of faces, and hence of any maximal chain of idempotents.

(ii) Let $S$ be a closed irreducible subsemigroup of $M$ containing $E(M)$. Then $S$ contains the neutral element of $M$; it follows that $S$ is a submonoid of $M$, and $G(S)$ is a subtorus of $T$. By (i), we have $\operatorname{dim}\left(T_{0}\right)=\operatorname{dim}\left(G(S)_{0}\right)$. But $G(S)_{0} \subset T_{0}$, and hence equality holds. Taking closures, we obtain that $S$ contains $\overline{T_{0}}$.

(iii) Let $S$ be a closed irreducible subsemigroup of $M$ having a zero, e. Then $e$ is idempotent; thus, $e e_{0}=e_{0}$. For any $x \in S$, we have $x e=e$ and hence $x e_{0}=e_{0}$. Also, $S$ is a toric monoid by Lemma 3.3. Thus, the closure $\overline{S T_{0}}$ is a toric monoid, stable by $T_{0}$ and contained in $M_{e_{0}}$ (the fiber of $\varphi$ at $e$ ). Since $T$ has finitely orbits in $M$, it follows that $T_{e_{0}}$ has finitely many orbits in $M_{e_{0}}$; since $T_{0}$ is a subgroup of finite index in $T_{e_{0}}$, we see that $T_{0}$ has finitely many orbits in $M_{e_{0}}$ as well. As a consequence, $\overline{S T_{0}}$ is the closure of a $T_{0}$-orbit, and thus equals $\overline{e_{S} T_{0}}$ for some idempotent $e_{S}$ of $M$. But $e_{S} \in \overline{T_{0}}$, and hence $\overline{S T_{0}} \subset \overline{T_{0}}$. In particular, $S \subset \overline{T_{0}}$.

(iv) It suffices to show that $T_{0}$ is defined over $F$. For this, we use the bijective correspondence between $F$-subgroup schemes of $T$ and $\Gamma$-stable subgroups of $\Lambda$, that associates with any such subgroup scheme $T^{\prime}$, the character group of the quotient torus $T / H$; then $T^{\prime}$ is a torus if and only if the corresponding subgroup $\Lambda^{\prime}$ is saturated in $\Lambda$, i.e., the quotient group $\Lambda / \Lambda^{\prime}$ is torsion-free (these results follow e.g. from [SGA3, Exp. VIII, $\S 2])$. Under this correspondence, the isotropy subgroup scheme $T_{e_{0}}$ is sent to the largest subgroup $\Lambda_{e_{0}}$ of $\Lambda$ contained in the monoid $\mathcal{M}$ (since $\mathcal{O}\left(T / T_{e_{0}}\right)=\mathcal{O}\left(e_{0} T\right)=\mathcal{O}\left(e_{0} M\right)$ is the subalgebra of $\mathcal{O}(M)$ generated by the invertible elements of that algebra). Thus, $T_{0}$ corresponds to the smallest saturated subgroup $\Lambda_{0}$ of $\Lambda$ that contains $\Lambda_{e_{0}}$. Clearly, the action of $\Gamma$ on $\Lambda$ stabilizes $\Lambda_{e_{0}}$, and hence $\Lambda_{0}$. 
Remark 3.5. The above proof can be shortened by using general structure results for unit dense algebraic monoids (see [Br12, §3.2]). We have chosen to present more self-contained arguments.

\subsection{The toric envelope}

In this subsection, we return to an irreducible commutative algebraic semigroup $S$; we denote by $e_{0}$ (resp. $e_{1}$ ) the smallest (resp. largest) idempotent of $S$, by $T$ the largest subtorus of $G\left(e_{1} S\right)$, and by $T_{0}$ the reduced neutral component of the isotropy subgroup scheme $T_{e_{0}}$. In view of Proposition $3.2, \bar{T}$ contains $E(S)$ and is the largest toric submonoid of $S$; we denote that submonoid by $T M(S)$ to emphasize its intrinsic nature. We now obtain an intrinsic interpretation of $\overline{T_{0}}$, thereby completing the proof of Theorem 1.2 .

Proposition 3.6. With the above notation, $\overline{T_{0}}$ is the smallest closed irreducible subsemigroup of $S$ containing $E(S)$, and also the largest toric subsemigroup of $S$ having a zero. Moreover, $\overline{T_{0}}$ is defined over $F$ if so is $S$.

Proof. Let $S^{\prime}$ be a closed irreducible subsemigroup of $S$ containing $E(S)$. Then we have $E(S) \subset T M\left(S^{\prime}\right) \subset T M(S)$ by Proposition [3.2. Thus, $T M\left(S^{\prime}\right)$ contains $\overline{T_{0}}$ in view of Proposition 3.4. Hence $S^{\prime} \supset \overline{T_{0}}$.

Next, let $M$ be a toric subsemigroup of $S$ having a zero. Then $M \subset T M(S)$ by Proposition 3.2 , and hence $M \subset \overline{T_{0}}$ by Proposition 3.4 again. The final assertion follows similarly from that proposition.

We denote $\overline{T_{0}}$ by $T E(S)$, and call it the toric envelope of $E(S)$; we may view $T E(S)$ as an algebro-geometric analogue of the finite poset $E(S)$.

Corollary 3.7. Let $S$ be an algebraic semigroup, $x$ a point of $S$, and $\langle x\rangle$ the smallest closed subsemigroup of $S$ containing $x$.

(i) $\langle x\rangle$ contains a largest closed toric subsemigroup, $T M(x)$.

(ii) $E(\langle x\rangle)$ is contained in a smallest closed irreducible subsemigroup of $\langle x\rangle$. Moreover, this subsemigroup, $T E(x)$, is a toric monoid.

(iii) $T M(x)=T M\left(x^{n}\right)$ and $T E(x)=T E\left(x^{n}\right)$ for any positive integer $n$.

(iv) If the algebraic semigroup $S$ and the point $x$ are defined over $F$, then so are $T M(x)$ and $T E(x)$.

Proof. (i) By [BrRe12, Lem. 1], there exists a positive integer $n$ such that $\left\langle x^{n}\right\rangle$ is irreducible. Since $\left\langle x^{n}\right\rangle$ is also commutative, it contains a largest closed toric subsemigroup by Proposition [3.2. But every toric subsemigroup $S$ of $\langle x\rangle$ is contained in $\left\langle x^{n}\right\rangle$. Indeed, we have $S^{n} \subset\left\langle x^{n}\right\rangle$; moreover, $S=S^{n}$, since the $n$th power map of $S$ restricts to a finite surjective homomorphism on any subtorus.

(ii) We claim that $E(\langle x\rangle)=E\left(\left\langle x^{n}\right\rangle\right)$ for any positive integer $n$. Indeed, $E\left(\left\langle x^{n}\right\rangle\right)$ is obviously contained in $E(\langle x\rangle)$. For the opposite inclusion, note that for any $y \in\langle x\rangle$, we have $y^{n} \in\left\langle x^{n}\right\rangle$. Taking $y$ idempotent yields the claim.

Now choose $n$ as in (i); then the desired statement follows from Proposition 3.6 in view of the claim.

(iii) is proved similarly; it implies (iv) in view of Propositions 3.2 and 3.6 again. 
Remark 3.8. When the algebraic semigroup $S$ is irreducible and commutative, we clearly have $T M(x) \subset T M(S)$ and $T E(x) \subset T E(S)$ for all $x \in S$. Moreover, if the field $k$ is not locally finite (that is, $k$ is not the algebraic closure of a finite field), then there exists $x \in S$ such that $T M(x)=T M(S)$ and $T E(x)=T E(S)$ : indeed, the torus $T=G(T M(S))$ has a point $x$ which generates a dense subgroup, and then $\langle x\rangle=T M(S)$.

On the other hand, if $k$ is locally finite, then any algebraic semigroup $S$ is defined over some finite subfield $\mathbb{F}_{q}$. Hence $S$ is the union of the finite subsemigroups $S\left(\mathbb{F}_{q^{n}}\right)$, where $n \geq 1$; it follows that $T M(x)=T E(x)$ consists of a unique point, for any $x \in S$.

Example 3.9. Let $S$ be a linear algebraic semigroup, i.e., $S$ is isomorphic to a closed subsemigroup of $\operatorname{End}(V)$ for some finite-dimensional vector space $V$. Given $x \in S$, we describe the combinatorial data of the toric monoid $T M(x)$ in terms of the spectrum of $x$ (viewed as a linear operator on $V$ ).

Consider the decomposition of $V$ into generalized eigenspaces for $x$,

$$
V=\bigoplus_{\lambda} V_{\lambda}
$$

where $\lambda$ runs over the spectrum. Since $x$ acts nilpotently on $V_{0}$ and $T M(x)=T M\left(x^{n}\right)$ for any positive integer $n$, we may assume that $x$ acts trivially on $V_{0}$. Then we may replace $V$ with $\bigoplus_{\lambda \neq 0} V_{\lambda}$, and hence assume that $x \in \mathrm{GL}(V)$. In that case, $\langle x\rangle \cap \mathrm{GL}(V)$ is a closed subsemigroup of the algebraic group $\mathrm{GL}(V)$, and hence a closed subgroup; in particular, $\operatorname{id}_{V} \in\langle x\rangle$. So $\langle x\rangle$ is a closed submonoid of $\operatorname{End}(V)$, and $G(\langle x\rangle)=\langle x\rangle \cap \mathrm{GL}(V)$. Thus, $T M(x)$ is the closure of the largest subtorus, $T$, of the commutative linear algebraic group $G:=G(\langle x\rangle)$.

In view of the Jordan decompositions $x=x_{s} x_{u}$ and $G=G_{s} \times G_{u}$, we see that $T$ is the largest subtorus of $G\left(\left\langle x_{s}\right\rangle\right)$. Thus, we may replace $x$ with $x_{s}$ and assume that

$$
x=\operatorname{diag}\left(\lambda_{1}, \ldots, \lambda_{r}\right),
$$

where $\lambda_{i} \in k^{*}$ for $i=1, \ldots, r$; we may further assume that $\lambda_{1}, \ldots, \lambda_{r}$ are pairwise distinct. Then $G(\langle x\rangle)$ is contained in the diagonal torus $\mathbb{G}_{m}^{r}$, and the character group of the quotient torus $\mathbb{G}_{m}^{r} / G(\langle x\rangle)$ is the subgroup of $\operatorname{Hom}\left(\mathbb{G}_{m}^{r}, \mathbb{G}_{m}\right) \cong \mathbb{Z}^{r}$ consisting of those tuples $\left(a_{1}, \ldots, a_{r}\right)$ such that $\prod_{i=1}^{r} \lambda_{i}^{a_{i}}=1$. Via the correspondence between closed subgroups of $\mathbb{G}_{m}^{r}$ and subgroups of $\mathbb{Z}^{r}$, it follows that the character group of $G(\langle x\rangle)$ is the subgroup of $k^{*}$ generated by $\lambda_{1}, \ldots, \lambda_{r}$. As a consequence, the free abelian group $\Lambda$ associated with the toric monoid $T M(x)$ is isomorphic to the subgroup of $k^{*}$ generated by the nth powers of the nonzero eigenvalues, for $n$ sufficiently divisible (so that this subgroup is indeed free).

Moreover, since the coordinate functions generate the algebra $\mathcal{O}(\langle x\rangle)$ and are eigenvectors of $G(\langle x\rangle)$, we see that the monoid $\mathcal{M}$ associated with $T M(x)$ is isomorphic to the submonoid of $(k, \times)$ generated by the $n$th powers of the eigenvalues, for $n$ sufficiently divisible (so that this monoid embeds indeed into a free abelian group).

Next, we describe the idempotents of $\langle x\rangle$, where $x$ is a diagonal matrix as above. These idempotents are among those of the subalgebra of End $(V)$ consisting of all diagonal matrices; hence they are of the form

$$
e_{I}:=\sum_{i \in I} e_{i}
$$


where $I \subset\{1, \ldots, r\}$, and $e_{i}$ denotes the projection to the $i$ th coordinate subspace. To determine when $e_{I} \in\langle x\rangle$, we view $\mathcal{M}$ as the monoid generated by $t_{1}, \ldots, t_{r}$, with relations of the form

$$
\prod_{a \in A} t_{i}^{a_{i}}=\prod_{b \in B} t_{j}^{b_{j}}
$$

where $A, B$ are disjoint subsets of $\{1, \ldots, r\}$ (one of them being possibly empty), and $a_{i}$, $b_{j}$ are positive integers; such relations will be called primitive. Since

$$
E(\langle x\rangle)=E(T M(x))=\operatorname{Hom}(\mathcal{M},\{1,0\}),
$$

it follows that $e_{I} \in\langle x\rangle$ if and only if either $I$ contains $A \cup B$, or $I$ meets the complements of $A$ and of $B$.

In particular, the largest idempotent of $\langle x\rangle$ is $e_{1, \ldots, r}=\mathrm{id}_{V}$ (this may of course be seen directly). The smallest idempotent is $e_{I}$, where $i \in I$ if and only if the $i$ th coordinate is invertible on $\langle x\rangle$; this is equivalent to the existence of a primitive relation of the form $\prod_{a \in A} t_{i}^{a_{i}}=1$, where $i \in A$.

\subsection{Algebraic semigroups with finitely many idempotents}

Consider an algebraic semigroup $S$ such that $E(S)$ is finite. Then $S$ has a smallest idempotent, as shown by the proof of Proposition 2.3 (ii). Also, when $S$ is irreducible, it has a largest idempotent by the proof of Proposition 3.1. We now obtain criteria for an idempotent of an algebraic semigroup to be the smallest or the largest one (if they exist):

Proposition 3.10. Let $S$ be an algebraic semigroup, and $e \in S$ an idempotent.

(i) $e$ is the smallest idempotent if and only if e is central and $e S$ is a group.

(ii) When $S$ is irreducible, $e$ is the largest idempotent if and only if $e$ is central and there exists a positive integer $n$ such that $x^{n} \in e S$ for all $x \in S$.

Proof. (i) Assume that $e$ is the smallest idempotent. Then both $e S_{e}$ and ${ }_{e} S e$ consist of the unique point $e$. Since $e$ is a minimal idempotent, it follows that $S e S=e S e$ by $[\mathrm{Br} 12$, Prop. 5 (ii)]. In particular, $x e \in e S e$ for any $x \in S$. As a consequence, $x e=e x e$; likewise, $e x=e x e$ and hence $e$ is central. Thus, $e S=e S e$; the latter is a group in view of [loc. cit., Prop. 4 (ii)].

To show the converse implication, let $f \in S$ be an idempotent. Then $e f$ is an idempotent of the group $e S$, and hence $e f=e=f e$.

(ii) If $e$ is the largest idempotent, then $e S_{e}$ and ${ }_{e} S e$ still consist of the unique point $e$. By Lemma 2.8, it follows that $e S e$ contains $U e$; likewise, $e S e$ contains $e U$. Since $S$ is irreducible, $e S e$ contains both $S e$ and $e S$. Arguing as in (i), this yields that $e$ is central. Also, there exists a positive integer $n$ such that every $x \in S$ satisfies $x^{n} \in e(x) S$ for some idempotent $e(x)$, by [BrRe12, Cor. 1]. Since $e(x) \leq e$, it follows that $x^{n} \in e S$.

For the converse implication, let again $f \in S$ be an idempotent. Then $f=f^{n} \in e S$, and hence $f=f e=e f$; in other words, $f \leq e$.

We now show that the structure of an irreducible algebraic monoid having finitely many idempotents reduces somehow to that of a closed irreducible submonoid having a zero and the same idempotents: 
Proposition 3.11. The following conditions are equivalent for an irreducible algebraic semigroup $S$ :

(i) $E(S)$ is finite.

(ii) $S$ has a smallest idempotent, $e_{0}$, and a largest one, $e_{1}$. Moreover, $E\left(M_{S}\right)$ is finite, where $M_{S}$ denotes the reduced neutral component of the submonoid scheme $e_{1} S_{e_{0}} \subset S$ (with unit $e_{1}$ and zero $e_{0}$ ).

Under either condition, $E(S)$ is reduced, central in $S$, and equals $E\left(M_{S}\right)$.

Proof. (i) $\Rightarrow$ (ii) follows from the discussion preceding Proposition 3.10. For the converse, note that $E\left(M_{S}\right)=E\left(e_{1} S_{e_{0}}\right)$ as sets, by the definitions of $e_{0}$ and $e_{1}$; also, $E\left(e_{1} S_{e_{0}}\right)=$ $E\left(M_{S}\right)$ as sets, in view of Theorem 2.14.

If $E(S)$ is finite, then it is reduced and central in $S$ by Proposition 2.10. Since $E(S)=E\left(M_{S}\right)$ as sets, it follows that this also holds as schemes.

The above reduction motivates the following statement, due to Putcha (see $\mathrm{Pu} 82$, Cor. 10]); we present an alternative proof, based on Renner's construction of the "largest reductive quotient" of an irreducible linear algebraic monoid.

Proposition 3.12. Let $M$ be an irreducible algebraic monoid having a zero. If $E(M)$ is finite, then $G(M)$ is solvable.

Proof. By [Re85, Thm. 2.5], there exist an irreducible algebraic monoid $M^{\prime}$ equipped with a homomorphism of algebraic monoids $\rho: M \rightarrow M^{\prime}$ that satisfies the following conditions: (i) $\rho$ restricts to a surjective homomorphism $G:=G(M) \rightarrow G\left(M^{\prime}\right)=$ : $G^{\prime}$ with kernel the unipotent radical, $R_{u}(G)$.

(ii) $\rho$ restricts to an isomorphism $\bar{T} \rightarrow \overline{T^{\prime}}$, where $T$ denotes a maximal subtorus of $G$, and $T^{\prime}$ its image under $\rho$.

As a consequence, $G^{\prime}$ is reductive, that is, $M^{\prime}$ is a reductive monoid. Also, since each conjugacy class of idempotents in $M$ (resp. $M^{\prime}$ ) meets $\bar{T}$ (resp. $\overline{T^{\prime}}$ ) and since every idempotent of $M$ is central, we see that $E\left(M^{\prime}\right)$ equals $E\left(\overline{T^{\prime}}\right)$ and is contained in the center of $M^{\prime}$.

Let $\mathcal{C}$ be the cone associated with the toric monoid $\overline{T^{\prime}}$. Then $\mathcal{C}$ is stable under the action of the Weyl group $W^{\prime}:=W\left(G^{\prime}, T^{\prime}\right)$ on the character group of $T^{\prime}$. Recall from Subsection 3.2 that $E\left(\overline{T^{\prime}}\right)$ is in a bijective correspondence with the set of faces of $\mathcal{C}$; this correspondence is compatible with the natural actions of $W^{\prime}$. But $W^{\prime}$ acts trivially on the idempotents of $\overline{T^{\prime}}$, since they are all central. Thus, $W^{\prime}$ stabilizes every face of $\mathcal{C}$. It follows that $W^{\prime}$ fixes pointwise every extremal ray; hence $W^{\prime}$ fixes pointwise the whole cone $\mathcal{C}$. Since the interior of $\mathcal{C}$ is nonempty, $W^{\prime}$ must be trivial, i.e., $G^{\prime}$ is a torus. Hence $G$ is solvable.

Acknowledgements. Most of this article was written during my participation in February 2013 to the Thematic Program on Torsors, Nonassociative Algebras and Cohomological Invariants, held at the Fields Institute. I warmly thank the organizers of the program for their invitation, and the Fields Institute for providing excellent working conditions. I also thank Mohan Putcha and Wenxue Huang for very helpful e-mail exchanges. 


\section{References}

[Br08] M. Brion, Local structure of algebraic monoids, Mosc. Math. J. 8, No. 4 (2008), 647-666.

[Br12] M. Brion, On algebraic semigroups and monoids, to appear in the proceedings of the Fields Institute workshop on Algebraic Monoids, Group Embeddings and Algebraic Combinatorics (July 3-6, 2012); available on arXiv:1208.0675.

[BrRi07] M. Brion and A. Rittatore, The structure of normal algebraic monoids, Semigroup Forum 74, No. 3 (2007), 410-422.

[BrRe12] M. Brion and L. Renner, Algebraic semigroups are strongly $\pi$-regular, to appear in the proceedings of the Fields Institute workshop on Algebraic Monoids, Group Embeddings and Algebraic Combinatorics (July 3-6, 2012); available on arXiv:1209.2042.

[Ha77] R. Hartshorne, Algebraic geometry, Graduate Texts in Mathematics 52, SpringerVerlag, New York, 1977.

[Hu96a] W. Huang, Nilpotent algebraic monoids, J. Algebra 179, No. 3 (1996), 720-731.

[Hu96b] W. Huang, Linear associative algebras with finitely many idempotents, Linear and Multilinear Algebra 40, No. 4 (1996), 303-309.

[Ne92] K.-H. Neeb, Toric varieties and algebraic monoids, Sem. Sophus Lie 2, No. 2 (1992), 159-187.

[Pu81] M. S. Putcha, On linear algebraic semigroups. III, Internat. J. Math. Math. Sci. 4, No. 4 (1981), 667-690; corrigendum, ibid., 5, No. 1 (1982), 205-207.

[Pu82] M. S. Putcha, The group of units of a connected algebraic monoid, Linear and Multilinear Algebra 12, No. 1 (1982), 37-50.

[Pu84] M. S. Putcha, Algebraic monoids with a dense group of units, Semigroup Forum 28, No 1-3 (1984), 365-370.

[Pu88] M. S. Putcha, Linear algebraic monoids, London Mathematical Society Lecture Note Series 133, Cambridge University Press, 1988.

[Ro56] M. Rosenlicht, Some basic theorems on algebraic groups, Amer. J. Math. 78 (1956), 401-443.

[Re85] L. E. Renner, Reductive monoids are von Neumann regular, J. Algebra 93, No. 2 (1985), 237-245.

[Re05] L. Renner, Linear algebraic monoids, Encyclopedia of Mathematical Sciences 134, Invariant Theory 5, Springer-Verlag, 2005. 
[Ri98] A. Rittatore, Algebraic monoids and group embeddings, Transform. Groups 3, No. 4 (1998), 375-396.

[Ri07] A. Rittatore, Algebraic monoids with affine unit group are affine, Transform. Groups 12, No. 3 (2007), 601-605.

[SGA3] Séminaire de Géométrie Algébrique du Bois-Marie 1962/64 (SGA3), Schémas en Groupes II, Lecture Notes Math. 152, Springer-Verlag, New York, 1970.

[Sp98] T. A. Springer, Linear algebraic groups. Second edition, Progress in Mathematics 9, Birkhäuser, Boston, MA, 1998.

Université de Grenoble I, Département de Mathématiques, Institut Fourier, UMR 5582, 38402 Saint-Martin d'Hères Cedex, France

E-mail address: Michel.Brion@ujf-grenoble.fr 